\title{
Influenza vaccination, pneumococcal vaccination and risk of acute myocardial infarction: matched case-control study
}

\author{
A. Niroshan Siriwardena PhD, Stella M. Gwini MSc, Carol A.C. Coupland CStat PhD
}

Previously published at www.cmaj.ca

\section{ABSTRACT}

Background: Previous studies have shown an association between acute myocardial infarction and preceding respiratory infection. Contradictory evidence exists on the influence of influenza vaccination and pneumococcal vaccination in preventing cardiovascular disease. We aimed to investigate the possible association of influenza vaccination and pneumococcal vaccination with acute myocardial infarction.

Methods: We used a matched case-control design with data from the United Kingdom General Practice Research Database. Cases were patients who were at least 40 years of age at diagnosis of first acute myocardial infarction recorded from Nov.1, 2001, to May 31, 2007, and were matched for sex, general practice, age and calendar time (i.e., month corresponding to index date of acute myocardial infarction), with up to four controls each. Data were analyzed using conditional logistic regression, adjusted for vaccination target groups, cardiovascular risk factors, treatment medications and attendances at a general practice.

Results: We included 78706 patients, of whom 16012 were cases and 62694 were matched controls. Influenza vaccination had been received in the previous year by 8472 cases $(52.9 \%)$ and 32081 controls (51.2\%) and was associated with a $19 \%$ reduction in the rate of acute myocardial infarction (adjusted odds ratio [OR] 0.81, 95\% confidence interval [Cl] 0.77-0.85). Early seasonal influenza vaccination was associated with a lower rate of acute myocardial infarction (adjusted OR 0.79, 95\% Cl 0.75-0.83) than vaccination after mid-November (adjusted OR $0.88,95 \% \mathrm{Cl}$ $0.79-0.97)$. Pneumococcal vaccination was not associated with a reduction in the rate of acute myocardial infarction (adjusted OR 0.96, 95\% Cl 0.91-1.02).

Interpretation: Influenza vaccination but not pneumococcal vaccination is associated with a reduced rate of first acute myocardial infarction. This association and the potential benefit of early seasonal vaccination need to be considered in future experimental studies.

$\mathrm{W}$ inter peaks in incidence of acute myocardial infarction have been linked to climate, ${ }^{1}$ metabolic factors $^{2}$ and infection. ${ }^{3}$ Because known risk factors do not fully account for cases of acute myocardial infarction, current interest is focused on the putative link with respiratory infection. Significant increases in acute myocardial infarction occur during peak winter incidence of pneumonia, influenza and influenza-like syndrome, ${ }^{4}$ particularly during years dominated by epidemic rather than nonepidemic influenza A. This association supports the notion that the increase is caused by influenza rather than cold weather. ${ }^{5}$

Acute myocardial infarction may increase susceptibility to respiratory illness, ${ }^{6}$ but the association between acute myocardial infarction and respiratory infection occurring within four weeks prior to the acute myocardial infarction ${ }^{7.8}$ supports infection as a cause of acute myocardial infarction. Although the exact mechanism is unknown, the favoured hypothesis is that infection triggers plaque rupture. ${ }^{9}$ Although several observational studies, as well as two randomized controlled trials, ${ }^{10,11}$ suggest a positive effect of influenza vaccine in preventing acute myocardial infarction, they provide insufficient and conflicting evidence. ${ }^{12}$

The aim of this study was to investigate a possible association between influenza or pneumococcal vaccination and acute myocardial infarction.

\section{Methods}

\section{Study design and ethics approval}

We used a matched case-control study design. Each case of acute myocardial infarction was matched to four controls according to age, sex, general practice attended and calendar time (i.e., month corresponding to index date of acute myocardial infarction), using incidence density sampling according to person-time at risk. ${ }^{13}$ The study was approved by the Independent Scientific Advisory Committee of the General Practice Research Database and the United Kingdom National Research Ethics Service.

\section{Data sources}

Data were extracted from the General Practice Research Database, an extensively validated computerized database, representative of and comprising 5\% of the population of England and Wales. ${ }^{14}$ Virtually all patients in the database are

From the Faculty of Health, Life and Social Sciences (Siriwardena), University of Lincoln and NHS Lincolnshire, Brayford Campus; NHS Lincolnshire (Gwini), Cross O'Cliff, Bracebridge Heath, Lincoln; and the Division of Primary Care (Coupland), School of Community Health Sciences, University of Nottingham, Nottingham, UK

CMAJ 2010. DOI:10.1503/cmaj.091891 
registered with a general practitioner, and all health care attendances are recorded in the database. The database contains reliable, anonymized patient data that includes demographic information, diagnoses, medication, health-related behaviours, referrals and treatment outcomes.

We estimated that 6176 cases and 24704 controls would provide $90 \%$ power to detect an odds ratio (OR) of 0.9 using a two-sided 5\% significance level, assuming $70 \%$ of controls were vaccinated and with a correlation coefficient of 0.2 between cases and matched controls for a clinically important effect based on current vaccination rates. ${ }^{15}$ All available cases in the General Practice Research Database were included in the study.

\section{Selection of cases and controls}

Case patients were at least 40 years of age at the time of diagnosis of first acute myocardial infarction (fatal or nonfatal) had clinical records for over five and a half years (between Nov. 1, 2001, to May 31, 2007) and were identified using standardized (Read and Oxford Medical Information Systems [OXMIS]) codes. The date of acute myocardial infarction, referred to as the index date, was used to match controls. Four controls were selected at random from the eligible matched controls for each case (i.e., before exposure status was known). We included only cases and controls with clinical records for at least five years before the index date to ensure completeness of recording of exposures and confounding variables.

\section{Identification of vaccination status}

Only data on influenza and pneumococcal vaccinations administered before the index date were extracted. For the main analyses, influenza vaccination was defined as vaccination given in the year preceding the index date. Other exposures to influenza vaccination considered were influenza vaccination within the current vaccination season (i.e., the same vaccination season as the index date) and early (i.e., between Sept. 1 and Nov. 15) or late vaccination (i.e., between Nov. 16 and Feb. 28 or 29, depending on the year).

Patients were considered to have had a pneumococcal vaccination if they had ever received the pneumococcal vaccine before the index date. Combined vaccination was defined as pneumococcal vaccination ever combined with influenza vaccination in the year preceding the index date.

\section{Confounding variables}

Data on potential confounding variables were extracted using Read and OXMIS codes recorded before the index date (Table 1). High-risk categories and target groups are identical for pneumococcal and influenza vaccination,,$^{15}$ as defined by the UK Department of Health (2008).

\section{Statistical analysis}

Conditional logistic regression was used to estimate unadjusted and adjusted ORs and 95\% confidence intervals (CIs) for the association between acute myocardial infarction and influenza and pneumococcal vaccination. Since we used incident patient cases who were matched with controls, were free of disease at the end of the study period and were from a dynamic population, ORs were taken as estimating rate ratios. ${ }^{13}$

The adjusted analyses accounted for confounders, including target groups for vaccination, additional cardiovascular risk factors, treatments and general practitioner consultations in the preceding five years, grouped into four categories based on quartiles of the distribution $(\leq 12,13-26,27-44$, and $\geq 45$ ). Smoking status was known for most patients $(92 \%)$ and included in the main analyses; patients whose smoking status was unknown were excluded. Each type of vaccination was adjusted for the other type.

Trends were established using likelihood ratio tests for trend. Subgroup analyses using likelihood ratio tests for interaction were conducted for age ( $<65$ and $\geq 65$ years) and vaccination target group (at least one target group present).

Systolic blood pressure, body mass index (BMI) and total cholesterol were not included in the main adjusted analyses owing to missing data $(63 \%, 45 \%$ and $15 \%$ completeness respectively). To replace missing values for smoking status, systolic blood pressure, BMI and total cholesterol, multiple imputation was conducted as an additional analysis, ${ }^{16}$ creating ten imputed data sets with results combined using Rubin's rules to account for uncertainty in the imputed data. ${ }^{17}$

\section{Results}

We included 16012 cases and 62964 matched controls from 379 practices. Most cases (95.7\%) had four controls. Confounding factors, apart from splenectomy and chronic liver disease, were reported more frequently in cases than controls (Table 1).

People with risk factors for acute myocardial infarction were more likely to be vaccinated (Table 2), which was expected since many cardiovascular risk factors are also indications for vaccination.

\section{Influenza vaccination and acute myocardial infarction}

Uptake of the influenza vaccine and results of the conditional logistic regression are shown in Table 3. The unadjusted analyses showed a slightly increased risk of acute myocardial infarction in vaccinated people. However, this analysis does not account for the fact that vaccinated people make up a preselected group and are already at increased risk for acute myocardial infarction because of their risk factors (unadjusted OR for acute myocardial infarction among people in a high-risk group is 2.26 (95\% CI 2.18 to 2.35 ).

After adjusting for confounding variables, so that vaccinated and unvaccinated groups were comparable in terms of risk factors in the model, we found that influenza vaccination within the previous year was associated with a significantly reduced rate of acute myocardial infarction (adjusted OR $0.81,95 \%$ CI 0.77 to $0.85, p<0.001$ ).

The confounders that contributed most to the change in OR for influenza vaccination were consultation rate, previous coronary heart disease and diabetes mellitus. Number of consultations before acute myocardial infarction was a key variable in the adjusted analysis; patients with more consultations were more likely to receive vaccination, but were also at increased risk of acute myocardial infarction - presumably 
Table 1: Characteristics of cases and controls

\begin{tabular}{|c|c|c|c|}
\hline \multirow[b]{2}{*}{ Characteristic } & \multicolumn{2}{|c|}{ No. $(\%) *$} & \multirow[b]{2}{*}{$\begin{array}{l}\text { Unadjusted OR } \\
\quad(95 \% \mathrm{Cl})\end{array}$} \\
\hline & $\begin{array}{c}\text { Cases } \\
n=16012\end{array}$ & $\begin{array}{c}\text { Controls } \\
n=62694\end{array}$ & \\
\hline \multicolumn{4}{|l|}{ Matching variables } \\
\hline \multicolumn{4}{|l|}{ Age, yr } \\
\hline $40-64$ & $5341(33.4)$ & $21359(34.1)$ & NAt \\
\hline$\geq 65$ & $10671(66.6)$ & 41335 (65.9) & NAt \\
\hline \multicolumn{4}{|l|}{ Sex } \\
\hline Male & $6168(38.5)$ & $24171(38.5)$ & NAt \\
\hline Female & $9844(61.5)$ & $38523(61.5)$ & NAt \\
\hline \multicolumn{4}{|l|}{ Vaccination target groups } \\
\hline Asthma or COPD attack, ever & $1980(12.4)$ & $5803(9.3)$ & $1.39(1.32-1.47)$ \\
\hline Chronic heart disease & $3756(23.5)$ & $6174(9.9)$ & $2.97(2.83-3.11)$ \\
\hline Stroke or transient ischemic attack & $1434(9.0)$ & $3487(5.6)$ & $1.66(1.56-1.78)$ \\
\hline Diabetes & $2451(15.3)$ & $5637(9.0)$ & $1.85(1.76-1.95)$ \\
\hline Splenectomy & $36(0.2)$ & $116(0.2)$ & $1.24(0.85-1.80)$ \\
\hline Chronic liver disease & $30(0.2)$ & $93(0.2)$ & $1.26(0.83-1.90)$ \\
\hline Chronic renal disease & $584(3.7)$ & $724(1.2)$ & $3.30(2.94-3.69)$ \\
\hline Immunosuppression or HIV & $2(0.01)$ & $3(<0.01)$ & $2.67(0.45-15.96)$ \\
\hline \multicolumn{4}{|l|}{ Other cardiovascular risk factors } \\
\hline Hyperlipidemia & $2180(13.6)$ & $5835(9.3)$ & $1.61(1.52-1.70)$ \\
\hline Family history of myocardial infarction & $302(1.9)$ & $954(1.5)$ & $1.29(1.12-1.48)$ \\
\hline BMI recorded within past $3 \mathrm{yr}$ & $8072(50.4)$ & $27434(43.8)$ & \\
\hline BMI kg/m², mean (SD) & $27.3(5.2)$ & $27.0(4.9)$ & $1.11 \neq(1.04-1.17)$ \\
\hline Peripheral vascular disease & $1216(7.6)$ & $2084(3.3)$ & $2.42(2.25-2.61)$ \\
\hline Hypertension & $6211(38.8)$ & $19538(31.2)$ & $1.45(1.40-1.51)$ \\
\hline \multicolumn{4}{|l|}{ Smoking history } \\
\hline Never smoked & $6292(39.3)$ & $31000(49.4)$ & 1.00 \\
\hline Ex-smoker & $4543(28.4)$ & $1545(25.1)$ & $1.49(1.42-1.56)$ \\
\hline Current smoker & $4265(26.7)$ & $10263(16.4)$ & $2.22(2.12-2.33)$ \\
\hline Not recorded & $912(5.7)$ & $5686(9.1)$ & \\
\hline Systolic blood pressure recorded in past yr & $11945(74.6)$ & $37338(59.6)$ & \\
\hline Systolic blood pressure $\mathrm{mmHg}$, mean (SD) & $140.5(21.4)$ & $141.4(18.0)$ & $0.98 \neq(0.96-0.99)$ \\
\hline Total cholesterol recorded & $2823(17.6)$ & $9150(14.6)$ & \\
\hline Total cholesterol mmol/L, mean (SD) & $5.37(1.27)$ & $5.28(1.13)$ & $1.04 \S(1.00-1.09)$ \\
\hline \multicolumn{4}{|l|}{ Treatments } \\
\hline Treatment with ASA ( $\geq 2$ prescriptions in previous $6 \mathrm{mo}$ ) & $1892(11.8)$ & $5211(8.3)$ & $1.52(1.44-1.62)$ \\
\hline Treatment with statins ( $\geq 1$ prescriptions in previous yr) & $1817(11.4)$ & $4372(7.0)$ & $1.79(1.69-1.90)$ \\
\hline Treatment with antihypertensives ( $\geq 1$ prescriptions in previous yr) & $1032(6.5)$ & $2468(3.9)$ & $1.72(1.59-1.85)$ \\
\hline \multicolumn{4}{|l|}{ No. of GP consultations in last 5 yr } \\
\hline$\leq 12$ & $3031(18.9)$ & $17196(27.4)$ & 1.00 \\
\hline $13-26$ & $3749(23.4)$ & $16618(26.5)$ & $1.37(1.30-1.45)$ \\
\hline 27-44 & $4193(26.2)$ & $15061(24.0)$ & $1.79(1.69-1.89)$ \\
\hline$\geq 45$ & $5039(31.5)$ & $13819(22.1)$ & $2.48(2.34-2.63)$ \\
\hline
\end{tabular}

Note: $\mathrm{ASA}=$ acetylsalicylic acid, $\mathrm{BMI}=$ body mass index, $\mathrm{Cl}=$ confidence interval, $\mathrm{COPD}=$ chronic obstructive pulmonary disease, GP = general practitioner, $\mathrm{NA}=$ not applicable, $\mathrm{OR}=$ odds ratio, SD = standard deviation.

*Unless otherwise indicated.

†Variables that were matched and hence not applicable.

$\neq$ Per 10-unit increase.

§Per unit increase. 
because they were sicker and hence consulting physicians more often. Adjusting for consultation rate alone resulted in an OR for vaccination of 0.84 (95\% CI 0.80 to 0.88 ). However, when we adjusted for all other confounders except consultations, influenza vaccination was still protective (OR 0.87 , 95\% CI 0.83 to 0.91 ).

When the association between acute myocardial infarction and influenza vaccination in the last year was further adjusted for "ever receiving pneumococcal vaccination," the result was an adjusted OR of 0.82 (95\% CI 0.78 to 0.86 ). Vaccination within the current vaccination season was also associated with a significantly reduced rate of acute myocardial infarction (adjusted OR $0.80,95 \%$ CI 0.76 to $0.84, p<0.001$ ). Most vaccinations administered were given early, between September and mid-November $(90.3 \%$ in cases and $91.0 \%$ in controls). When compared with no vaccination, early vaccination was associated with a $21 \%$ reduction in the rate of acute myocardial infarction, whereas late vaccination was associated with a $12 \%$ reduction. Compared with late vaccination, early vaccination was associated with a significantly reduced rate of acute myocardial infarction (adjusted OR $0.90,95 \%$ CI 0.82 to $1.00, p=0.042$ ). Influenza vaccination was associated with a significantly reduced rate of acute myocardial infarction in all periods. There was no significant reduction in acute myocardial infarction when the last vaccination was given more than a year before the index date.
Repeated vaccination (i.e., vaccination in all previous five or six consecutive seasons) was associated with a significant reduction in the rate of acute myocardial infarction (adjusted OR $0.79,95 \%$ CI 0.74 to 0.84 ) compared with no vaccinations in the previous five years, as was vaccination within the current season (OR $0.85,95 \%$ CI 0.80 to 0.90 ), but not consecutively in the five or six preceding vaccination seasons.

We conducted separate analyses and tests for interaction according to vaccination target group (i.e., with at least one target group present). The association between influenza vaccination and acute myocardial infarction was more marked for those in a vaccination target group (adjusted OR $0.70,95 \% \mathrm{CI} 0.64$ to 0.77) compared with those not in a target group (adjusted OR $0.85,95 \%$ CI 0.79 to 0.91 , test for interaction $p<0.001$ ).

In the analyses using multiply imputed data with BMI, systolic blood pressure and total cholesterol added to the covariates, the adjusted OR for influenza vaccination in the previous year was 0.83 (95\% CI 0.80 to 0.88 ).

\section{Pneumococcal vaccination and acute myocardial infarction}

Just over one-third of cases (35.4\%) and controls (34.7\%) had received a pneumococcal vaccination before the index date (Appendix 1, available at www.cmaj.ca/cgi/content/full/cmaj $.091891 / \mathrm{DC} 1$ ), and almost all of these (27 257 of 27 887) had been vaccinated within 10 years of the index date.

Table 2: Distribution of risk factors for acute myocardial infarction by vaccination

\begin{tabular}{|c|c|c|c|c|}
\hline Risk factor & \multicolumn{4}{|c|}{ No. $(\%) *$} \\
\hline Chronic heart disease & $2026(5.8)$ & $2111(13.6)$ & $586(20.1)$ & 5207 (20.9) \\
\hline Stroke or TIA & $1103(3.1)$ & $1150(7.4)$ & $268(9.2)$ & $2400(9.6)$ \\
\hline Chronic liver disease & $43(0.1)$ & $25(0.2)$ & $12(0.4)$ & $43(0.2)$ \\
\hline Chronic renal disease & $240(0.7)$ & $245(1.6)$ & $118(4.1)$ & $705(2.8)$ \\
\hline Splenectomy & $22(0.1)$ & $3(0.02)$ & $30(1.0)$ & $97(0.4)$ \\
\hline Hypertension & $7345(20.8)$ & $5822(37.4)$ & $1203(41.4)$ & $11379(45.6)$ \\
\hline Obesity or overweight & $7900(22.4)$ & $4595(29.5)$ & 999 (34.3) & $9689(38.8)$ \\
\hline $\begin{array}{l}\text { Treatment with } \\
\text { antihypertensives }\end{array}$ & $949(2.7)$ & $672(4.3)$ & $173(6.0)$ & $1706(6.8)$ \\
\hline Treatment with statins & $1486(4.2)$ & $1263(8.1)$ & $319(11.0)$ & $3121(12.5)$ \\
\hline Treatment with ASA & $1601(4.5)$ & $1458(9.4)$ & $350(12.0)$ & $3694(14.8)$ \\
\hline Family history of AMI & $531(1.5)$ & $261(1.7)$ & $43(1.5)$ & $421(1.7)$ \\
\hline $\begin{array}{l}\text { Average no. GP consultations in } \\
\text { the past } 5 \mathrm{yr} \text {, mean }(95 \% \mathrm{Cl})\end{array}$ & $19.3(19.1-19.5)$ & $36.3(35.9-36.7)$ & $41.1(40.0-42.2)$ & $44.9(44.6-45.3)$ \\
\hline
\end{tabular}

Note: $\mathrm{AMI}=$ acute myocardial infarction, $\mathrm{ASA}=$ acetylsalicylic acid, $\mathrm{Cl}=$ confidence interval, $\mathrm{COPD}=$ chronic obstructive pulmonary disease, GP = general practitioner, TIA = transient ischemic attack.

*Unless otherwise indicated. 
When adjusted for all covariates including influenza vaccination, there was no significant effect of pneumococcal vaccination (adjusted OR $0.96,95 \%$ CI 0.91 to 1.02 ). Using multiply imputed data and also adjusting for BMI, systolic blood pressure and total cholesterol, the adjusted OR for pneumococcal vaccination was 0.98 (95\% CI 0.93 to 1.04 ). Combined influenza and pneumococcal vaccination did not show significant benefit when compared with influenza vaccination alone (adjusted OR $0.94,95 \%$ CI 0.89 to $1.01, p=0.07$ ).

\section{Interpretation}

Influenza vaccination within the past year was associated with a $19 \%$ reduction in the rate of acute myocardial infarction among patients aged 40 years and over. Influenza vaccination administered within influenza season was also associated with a significant reduction (20\%) in the rate of acute myocardial infarction. We found that early vaccination within the influenza season (i.e., September to mid-November) was associated with greater benefit than vaccination later in the season $(21 \%$ v. $12 \%$ reduction in the rate of acute myocardial infarction compared with no vaccination). Pneumococcal vaccination or combined vaccination had no additional benefit compared with influenza vaccination alone.

Unsurprisingly, cases and controls were different in their disease and treatment characteristics, with cases more likely to have comorbidities or be receiving treatment than controls. Cases were more likely to have been vaccinated than controls, particularly those between age 40 and 64 years, a finding that accords with current UK guidelines to vaccinate all those in a target group. Importantly, we found that multivariate adjustment for confounding factors led to a protective association for influenza vaccination.

Convincing evidence of a protective role for influenza infection is emerging. Our findings lend support to previous evidence from observational studies of primary prevention ${ }^{10}$ and randomized studies of secondary prevention ${ }^{11}$ suggesting a beneficial effect of influenza vaccination for prevention of acute myocardial infarction or its complications. Previous observational studies have been limited by methodologic problems, including recall bias in self-reported vaccination

Table 3: Association between influenza vaccination and acute myocardial infarction

\begin{tabular}{|c|c|c|c|c|c|}
\hline Characteristic & \multicolumn{2}{|c|}{ No. $(\%)$} & \multicolumn{3}{|c|}{ OR $(95 \% \mathrm{Cl})$} \\
\hline \multicolumn{6}{|c|}{ Vaccination in preceding yr } \\
\hline All ages ( $\geq 40 \mathrm{yr}$ ) & $8472(52.9)$ & $32081(51.2)$ & $1.08(1.04-1.13)$ & $0.81(0.77-0.85)$ & $0.83(0.80-0.88)$ \\
\hline$<65 \mathrm{yr}$ & $1035(19.4)$ & $3166(14.8)$ & $1.41(1.30-1.53)$ & $0.81(0.73-0.90)$ & $0.83(0.75-0.92)$ \\
\hline \multicolumn{6}{|c|}{$\begin{array}{l}\text { Time since last vaccination at } \\
\text { index date, mo }\end{array}$} \\
\hline Never vaccinated & $5842(36.5)$ & $25423(40.6)$ & 1.00 & 1.00 & $1.00(0.78-0.90)$ \\
\hline $0-3$ & 3187 (19.9) & $12395(19.8)$ & $1.16(1.09-1.24)$ & $0.80(0.74-0.86)$ & $0.84(0.80-0.94)$ \\
\hline $3-6$ & $2230(13.9)$ & 8354 (13.3) & $1.22(1.13-1.32)$ & $0.82(0.76-0.89)$ & $0.86(0.85-0.99)$ \\
\hline \multicolumn{6}{|c|}{ Within-season vaccination } \\
\hline Yes & $7496(46.8)$ & $28487(45.4)$ & $1.07(1.02-1.12)$ & $0.80(0.76-0.84)$ & $0.83(0.79-0.87)$ \\
\hline $\begin{array}{l}\text { Early within-season } \\
\text { (Sept. to mid-Nov.) }\end{array}$ & $6770(42.3)$ & $25911(41.3)$ & $1.07(1.01-1.11)$ & $0.79(0.75-0.83)$ & $0.82(0.78-0.86)$ \\
\hline $\begin{array}{l}\text { Late within-season } \\
\text { (mid-Nov. to Feb.) }\end{array}$ & $726 \quad(4.5)$ & $2571(4.1)$ & $1.16(1.05-1.27)$ & $0.88(0.79-0.97)$ & $0.90(0.82-1.00)$ \\
\hline \multicolumn{6}{|c|}{$\begin{array}{l}\text { Vaccination in previous yr, by } \\
\text { mo of index date }\end{array}$} \\
\hline Sept. to Nov. & $1744(46.8)$ & $6862(47.0)$ & $0.97(0.89-1.06)$ & $0.75(0.68-0.83)$ & $0.77(0.70-0.85)$ \\
\hline Dec. to Mar. & $3418(55.6)$ & $12761(53.1)$ & $1.13(1.06-1.21)$ & $0.86(0.79-0.93)$ & $0.88(0.82-0.95)$ \\
\hline
\end{tabular}

Note: $\mathrm{Cl}=$ confidence interval, $\mathrm{OR}=$ odds ratio.

*Adjusted for asthma or chronic obstructive pulmonary disease, chronic heart disease, stroke or transient ischemic attack, diabetes, splenectomy, chronic liver disease, chronic renal failure, immunosuppression and HIV, hyperlipidemia, family history of acute myocardial infarction, peripheral vascular disease, hypertension, smoking status, treatment with acetylsalicylic acid, treatment with statins, treatment with antihypertensives, and general practice consultations. tAdjusted for all of the above variables as well as for body mass index, systolic blood pressure and total cholesterol using 10 multiply imputed data sets.

¥Totals for the period of Sept. to Nov. were 3723 cases and 14599 controls. Totals for the period of Dec. to Mar. were 6146 cases and 24036 controls. Totals for the period of Apr. to Aug. were 6143 cases and 24059 controls. 
status. ${ }^{10,18}$ Studies with no evidence of benefit had low power, poor case ascertainment, misclassification of vaccination status and lack of investigator blinding. ${ }^{19-21}$ The additional benefit of early within-season influenza vaccination (i.e., during the period from September to mid-November) compared with late vaccination (i.e., after mid-November) has been suggested by findings of previous research, ${ }^{22}$ but is not part of current recommendations for influenza vaccination..$^{23}$

Given that influenza vaccination is likely to be effective only against circulating strains of influenza virus, our findings are strengthened by the biological plausibility of a within-season or within-year effect of influenza vaccination in preventing influenza-related acute myocardial infarction. The finding that influenza vaccination was associated with a reduction in the risk of acute myocardial infarction, whereas no effect was found for pneumococcal vaccination, is evidence against the explanation that unknown confounders constituted a "healthy user" effect. If the 12-month protective effect of influenza vaccination was a result of confounding by indication, then we would also expect to find a protective effect for influenza vaccination beyond 12 months and for pneumococcal vaccination by the same token. But we did not. We believe that this discrepancy also supports the plausibility of our findings, and that the difference between the effect of early versus late vaccination on the risk of acute myocardial infarction cannot be explained by confounding by indication. Influenza vaccination was beneficial regardless of the month of acute myocardial infarction. This finding could be an indication of longerterm benefits for prevention of influenza infection, given that vaccinations considered in this instance had been given in the preceding 12 months. Following biological explanations for the association between acute myocardial infarction and influenza, the flu virus would have had a lesser effect on the vaccinated patients' circulatory systems.

Although previous studies have shown an additive effect of pneumococcal vaccination for prevention of respiratory disease,${ }^{24}$ we did not find a reduction in the rate of acute myocardial infarction for pneumococcal vaccination after adjusting for influenza vaccination, even among people aged 65 years and over. Our findings contrast with those of a recent study suggesting that pneumococcal vaccination was associated with a $50 \%$ reduction in the rate of acute myocardial infarction. ${ }^{25}$ Investigators in that study did not account for differences in cardiovascular risk factors, nor, crucially, for the confounding effect of influenza vaccination.

\section{Strengths and limitations}

Despite their known problems of bias and confounding, casecontrol designs are efficient in examining the association between outcomes and exposures. The large General Practice Research Database sample provided good power to investigate associations in a representative population with precision. We minimized selection bias by including all cases of acute myocardial infarction within the selected time period and matched controls, free of the outcome of interest and independent of the exposure of interest. ${ }^{26}$ Matching for age, sex, practice and calendar time (month corresponding to index date of acute myocardial infarction) increased the preci- sion of our results compared with those of previous, unmatched case-control studies. Information on exposures was recorded before the index date, eliminating recall bias.

To minimize bias due to misclassification and missing data, patients were selected only if they had at least five years of up-to-standard data on the General Practice Research Database. To reduce confounding from "healthy user," "indication" or "treatment" biases, we adjusted for many known confounders, including vaccination target groups and cardiovascular risk groups, treatments and general practitioner consultation rate, of which the latter is thought to be related to functional and economic status. ${ }^{27}$ Potential confounders such as functional status were not fully accounted for because of unavailability of data, and we did not have data for other triggers, such as stressful life events. Although we observed missing values for variables such as smoking status, systolic blood pressure, BMI and total cholesterol, we used standard imputation procedures to account for these and obtained results similar to those of our complete case analysis.

\section{Conclusion}

Our findings reinforce current recommendations for annual influenza vaccination of target groups, ${ }^{23,28}$ with a potential added benefit for prevention of acute myocardial infarction in those without established cardiovascular disease. ${ }^{29}$ This benefit may lead to an increase in suboptimal rates of vaccination, particularly among younger patients..$^{15}$

Further research is needed to confirm our finding that early vaccination, in particular, confers additional reduction in risk for acute myocardial infarction. If substantiated, this finding has implications for timely supply and administration of influenza vaccine and could lead to changes in recommendations for timing of vaccination.

This article has been peer reviewed.

Competing interests: None declared.

Contributors: Niroshan Siriwardena had the initial idea for the study and received support in the conception and design of the study from Carol Coupland. Stella Gwini was involved in the design of the study, performed the analysis of the data supported by Carol Coupland, and wrote the first draft of the article. All of the authors interpreted the results, participated in reviewing and revising the manuscript, and approved the final version of the manuscript submitted for publication.

Acknowledgements: The authors thank Prof. Tom Meade and Mr. Tim Clayton of the London School of Hygiene and Tropical Medicine for their support and advice, and the General Practice Research Database for providing data for the study.

Funding: This study was supported by funding from the Research for Patient Benefit Program of the National Institute for Health Research, United Kingdom [PB-PG-0706-10449] and was conducted independently from the funding body. The sponsor had no role in the design of the study, the collection, analysis or interpretation of the data, the writing of the manuscript or the decision to submit the study for publication. Niroshan Siriwardena had full access to all of the data and final responsibility for the decision to submit the study for publication.

\section{REFERENCES}

1. The Eurowinter Group. Cold exposure and winter mortality from ischaemic heart disease, cerebrovascular disease, respiratory disease, and all causes in warm and cold regions of Europe. Lancet 1997;349:1341-6. 
2. Mavri A, Guzic-Salobir B, Salobir-Pajnic B, et al. Seasonal variation of some metabolic and haemostatic risk factors in subjects with and without coronary artery disease. Blood Coagul Fibrinolysis 2001;12:359-65.

3. Danesh J, Whincup P, Walker M, et al. Low grade inflammation and coronary heart disease: prospective study and updated meta-analyses. BMJ 2000;321:199-204.

4. Dobson AJ, Alexander HM, Al-Roomi K, et al. Coronary events in the Hunter region of New South Wales, Australia: 1984-1986. Acta Med Scand Suppl 1988;728:84-9.

5. Reichert TA, Simonsen L, Sharma A, et al. Influenza and the winter increase in mortality in the United States, 1959-1999. Am J Epidemiol 2004;160:492-502.

6. Pell JP, Cobbe SM. Seasonal variations in coronary heart disease. QJM 1999; 92:689-96.

7. Spodick DH, Flessas AP, Johnson MM. Association of acute respiratory symptoms with onset of acute myocardial infarction: prospective investigation of $150 \mathrm{consec}-$ utive patients and matched control patients. Am J Cardiol 1984;53:481-2.

8. Penttinen J, Valonen P. The risk of myocardial infarction among Finnish farmer seeking medical care for an infection. Am J Public Health 1996;86:1440-2.

9. Harskamp RE, van Ginkel MW. Acute respiratory tract infections: a potential trigger for the acute coronary syndrome. Ann Med 2008;40:121-8.

10. Naghavi M, Barlas Z, Siadaty S, et al. Association of influenza vaccination and reduced risk of recurrent myocardial infarction. Circulation 2000;102:3039-45.

11. Gurfinkel EP, Leon de la FR, Mendiz O, et al. Flu vaccination in acute coronary syndromes and planned percutaneous coronary interventions (FLUVACS) Study. Eur Heart J 2004;25:25-31.

12. Keller T, Weeda VB, van Dongen CJ, et al. Influenza vaccines for preventing coronary heart disease [review]. Cochrane Database Syst Rev 2008;(3):CD005050.

13. Knol MJ, Vandenbroucke JP, Scott P, et al. What do case-control studies estimate? Survey of methods and assumptions in published case-control research. Am J Epidemiol 2008;168:1073-81

14. Jick SS, Kaye JA, Vasilakis-Scaramozza C, et al. Validity of the general practice research database. Pharmacotherapy 2003;23:686-9.

15. Department of Health. The influenza immunisation programme 3rd ed. London (UK): The Department; 2008. p. 1-15.

16. Royston P. Multiple imputation of missing values: update of ICE. Stata J 2005; 5:527-36.

17. Rubin DB. Multiple imputation for nonresponse in surveys. Hoboken (NJ): WileyInterscience; 2004.

18. Siscovick DS, Raghunathan TE, Lin D, et al. Influenza vaccination and the risk of primary cardiac arrest. Am J Epidemiol 2000;152:674-7.

19. Jackson LA, Yu O, Heckbert SR, et al. Influenza vaccination is not associated with a reduction in the risk of recurrent coronary events. Am J Epidemiol 2002;156:634-40.

20. Heffelfinger JD, Heckbert SR, Psaty BM, et al. Influenza vaccination and risk of incident myocardial infarction. Hum Vaccin 2006;2:161-6.

21. Meyers DG, Beahm D, Jurisich PD, et al. Influenza and pneumococcal vaccinations fail to prevent myocardial infarction. HeartDrug 2004;4:96-100.

22. Chodick G, Heymann AD, Green MS, et al. Late influenza vaccination is associated with reduced effectiveness. Prev Med 2006;43:71-6.

23. Fiore AE, Shay DK, Broder K, et al. Prevention and control of influenza: recommendations of the Advisory Committee on Immunization Practices (ACIP), 2008 MMWR Recomm Rep 2008;57(RR-7):1-60.

24. Christenson B, Hedlund J, Lundbergh P, et al. Additive preventive effect of influenza and pneumococcal vaccines in elderly persons. Eur Respir J 2004;23:363-8

25. Lamontagne F, Garant MP, Carvalho JC, et al. Pneumococcal vaccination and risk of myocardial infarction. CMAJ 2008;179:773-7.

26. Grimes DA, Schulz KF. Compared to what? Finding controls for case-control studies. Lancet 2005:365:1429-33.

27. Jordan K, Ong BN, Croft P. Previous consultation and self reported health status as predictors of future demand for primary care. J Epidemiol Community Healt 2003;57:109-13.

28. Begg NT, Salisbury DM. Immunisation against infectious diseases. London (UK): HMSO; 1996

29. Warren-Gash C, Smeeth L, Hayward AC. Influenza as a trigger for acute myocardial infarction or death from cardiovascular disease: a systematic review. Lance Infect Dis 2009;9:601-10.

Correspondence to: Prof. A. Niroshan Siriwardena, Faculty of Health, Life and Social Sciences, University of Lincoln, Lincoln LN6 7TS, UK; nsiriwardena@lincoln.ac.uk

\section{HYDROMORPH Contin'qi2h}

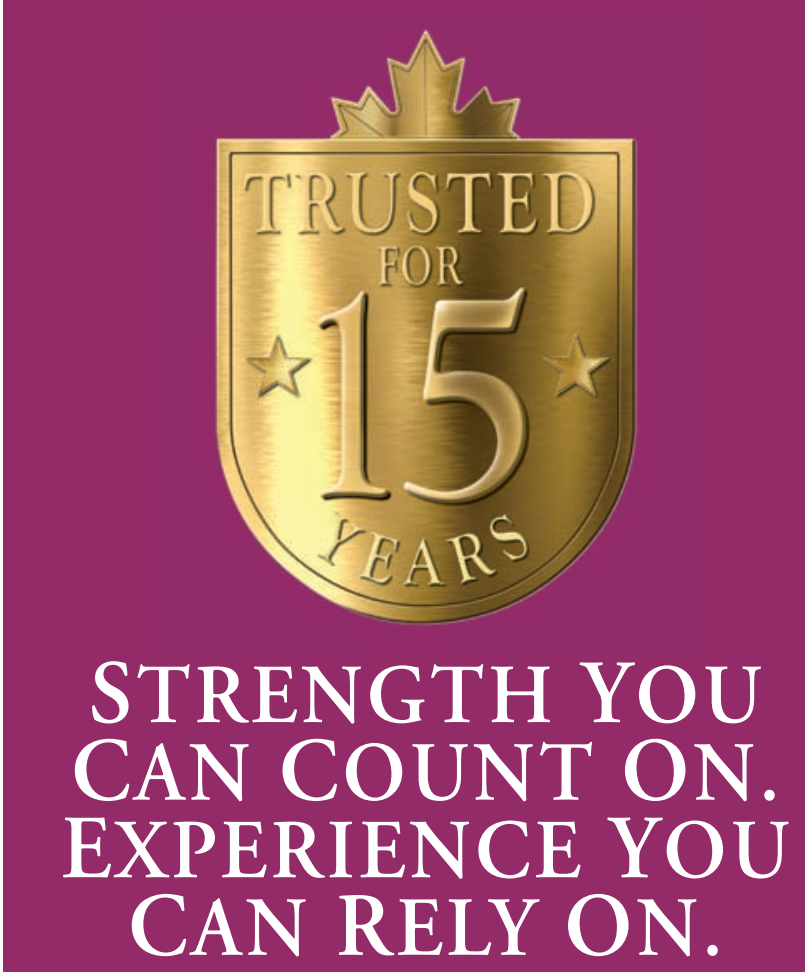

Hydromorph Contin ${ }^{\circledR}$ is indicated for the relief of severe chronic pain requiring the prolonged use of an oral opioid preparation.

Side effects are similar to other opioid analgesics. The most frequently observed are asthenic conditions, confusion, constipation, dizziness, lightheadedness, nausea, sedation, sweating and vomiting. Dosage limitations may be imposed by adverse effect. If they occur, please refer to prescribing information.

Warning: Opioid analgesics should be prescribed and handled with a high degree of caution appropriate to the use of a drug with strong abuse potential. Patients should be cautioned not to consume alcohol while taking Hydromorph Contin ${ }^{\circledR}$, as it may increase the chance of experiencing dangerous side effects. Hydromorph Contin ${ }^{\circledR} 18 \mathrm{mg}$ capsules and higher are for use in opioid tolerant patients only. There is a potential for fatal respiratory depression in patients not previously exposed to similar equianalgesic doses of an opioid analgesic. Hydromorph Contin ${ }^{\circledR}$ capsules or capsule beads should not be chewed, crushed or dissolved since this can lead to rapid release and absorption of a potentially fatal dose of hydromorphone. Product monograph available on request.

Hydromorph Contin ${ }^{\circledR}$ capsule beads may be sprinkled on cold, soft food.

\section{'HYDROMORPH Contin q12h \\ Controlled release hydromorphone capsules}

A trusted choice for severe chronic pain

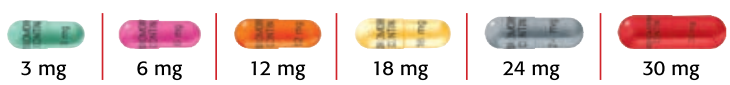

Initiation at $3 \mathrm{mg} \mathrm{q} 12 \mathrm{~h}$ or calculate the approximate daily oral hydromorphone dosage that should provide equivalent analgesia. See Product Monograph Table 1, Opioid Analgesics:

Approximate Analgesic Equivalences. 